\title{
Approprié signifie approprié!
}

\section{Urs Stoffel}

Dr méd., membre du Comité central de la FMH, responsable du département Médecine et tarifs ambulatoires

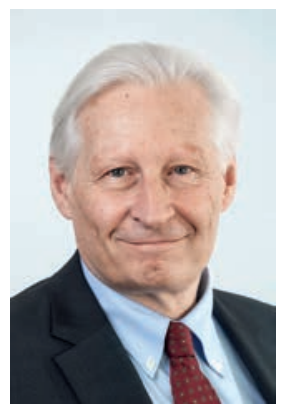

La consultation relative à l'intervention du Conseil fédéral dans la structure tarifaire TARMED s'est achevée le 21 juin 2017. Elle a donné lieu à une pléthore de prises de position indignées et scandalisées remises à l'OFSP. Le corps médical n'a pas été le seul, d'autres organisations se sont également offusquées des mesures du Conseil fédéral et ont manifesté leur inquiétude. Les critiques et la colère du corps médical ont été largement relayées par la presse. Aussi bien les médias que les politiques n'ont cessé de répéter que les médecins ne pouvaient s'en prendre à personne, c'était de leur faute parce qu'ils n'avaient pas réussi à s'entendre avec les partenaires tarifaires pour remettre une structure tarifaire révisée dans les temps au Conseil fédéral. L'impatience et l'incompréhension qui ressortent de ces déclarations sont indéniables mais aussi en partie compréhensibles.

\section{Un arrêt aux conséquences importantes}

L'arrêt du Tribunal arbitral du canton de Lucerne, publié le 29 mai 2017 au beau milieu de la consultation, a suscité beaucoup d'attention: cet arrêt analyse la première intervention tarifaire du Conseil fédéral du 20 juin 2014 du point de vue de sa légalité. Il arrive à la conclusion (sans être encore entré en force) que cette première intervention n'est pas légale parce qu'elle ne respecte pas le principe selon lequel le tarif doit être fixé «d'après les règles applicables en économie d'entreprise et structuré de manière appropriée» (art. 43 al. 4 LAMal).

Si le Conseil fédéral intervient dans la structure tarifaire, il doit rendre au tarif son caractère approprié.

Dans les considérants, le Tribunal rappelle que le Conseil fédéral, conformément à l'art. 43 al. 5bis LAMal, peut faire valoir sa compétence subsidiaire pour procéder à des adaptations de la structure tarifaire si celle-ci s'avère inappropriée et que les parties ne peuvent s'entendre sur une révision de la structure. Parallèlement, il souligne qu'une telle intervention doit rendre au tarif son caractère approprié. Par conséquent, l'intervention tarifaire est elle aussi soumise aux dispositions lé- gales de l'art. 43 al. 4 LAMal. Aucun tribunal ne s'était encore jamais prononcé sur la manière d'intervenir dans un tarif et à quelles dispositions légales une telle intervention devait répondre.

Si, en comparaison, l'intervention de 2014 était encore simple et limitée, celle de cette année est si complexe qu'elle risque en partie de provoquer des déséquilibres et des distorsions importantes. Les données et les faits

TARCO: seule une révision globale mène à une structure tarifaire ambulatoire appropriée et conforme aux principes d'économie d'entreprise.

qui motivent les différentes mesures proposées font tout simplement défaut. Dans ce contexte, on peut raisonnablement prétendre que cette deuxième intervention du Conseil fédéral ne répondra pas aux dispositions légales de l'art. 43 al. 4 LAMal.

\section{Le projet TARCO suit son cours}

La FMH a toujours déclaré clairement que les interventions ponctuelles pour corriger une structure tarifaire TARMED qui n'est plus à jour ne feront qu'accentuer les déséquilibres et la rendre encore moins appropriée. Pour la FMH, la seule voie pertinente consiste à poursuivre la révision globale du TARMED en concertation avec les partenaires tarifaires et dans le respect du caractère approprié et conforme aux principes de l'économie d'entreprise, énoncé dans la loi.

Le projet TARCO suit son cours: les 30 groupes de travail examinent les différents chapitres qui leur sont attribués. Certains chapitres sont déjà en consultation interne à la FMH. Le principal organe de décision mis en place par l'Assemblée des délégués de la FMH, le "TARCO COCKPIT», composé de différents représentants des organisations faîtières, se réunit régulièrement et il a déjà pris plus de 40 décisions.

Lors de la prochaine phase, nous approcherons successivement les partenaires tarifaires en vue des négociations communes.

Notre but reste de garantir le caractère approprié au travers d'une révision globale du TARMED en concertation avec nos partenaires tarifaires. 